\title{
Permanencia estudiantil: Factores que inciden en el Politécnico Internacional de Bogotá, Colombia
}

\author{
Student Permanence: Factors That Influence at Politecnico Internacional of Bogota, Colombia
}

\section{Permanência estudantil: factores que coincidem no politécnico internacional de Bogotá, Colombia}

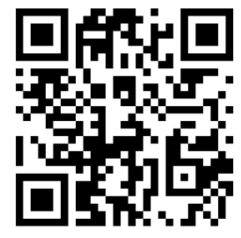

Corregido • Revised • Revisado: 11 / 08 / 2020

Aceptado • Accepted • Aprovado: 23 / 11 / 2020

\begin{abstract}
Resumen: El presente trabajo se centra en la identificación de factores relacionados con la permanencia estudiantil en educación superior. Fue llevado a cabo en la Institución de Educación Superior Politécnico Internacional (Bogotá, Colombia). Con un planteamiento metodológico descriptivo se recolectaron datos con la utilización de cuestionarios ad-hoc, con una muestra de 1487 estudiantes. Los resultados evidencian la importancia de las variables calidad y perfil del programa ofertado, en lo relacionado con la retención estudiantil. Se observa la influencia de factores motivacionales y de satisfacción personal sobre la persistencia del estudiantado dentro del programa. Las variables retención y persistencia son caracterizadas en el presente estudio como procesos independientes, susceptibles de estudio, que interactúan e inciden directamente en la permanencia en la educación superior.
\end{abstract}

Palabras claves: Abandono escolar; retención; educación superior; educación técnica; deserción escolar. 
http://doi.org/10.15359/ree.25-1.6

http://www.una.ac.cr/educare

educare@una.ac.cr

\begin{abstract}
This paper focuses on the identification of factors related to students' retention. This study was conducted at Politécnico Internacional (Bogotá, Colombia), a private higher education institution. To collect the data, some ad hoc surveys were administered to a sample of 1487 students. Concerning the student's retention, results highlight the importance of variables such as the quality and profile of the academic program offered. The influence of motivational factors and personal satisfaction about the students' persistence within the academic program is also observed. These two variables, retention and persistence, are characterized in this paper as independent processes, susceptible to study, which interact and have a direct effect on retention in higher education.
\end{abstract}

Keywords: dropout, retention, higher education, technical education

Resumo: Este artigo está focado na identificação de fatores relacionados à permanência estudantil no ensino superior. Este estudo foi realizado em uma Instituição de Ensino Superior chamada Politécnico Internacional (Bogotá, Colômbia). Com a opção metodológica descritiva, foram coletados os dados, utilizando o questionário ad-hoc, com uma amostra de 1487 estudantes. Os resultados indicam a importância de variáveis como qualidade e perfil do programa acadêmico oferecido, no que se refere a permanência estudantil. Observa-se também a influência de fatores motivacionais e a satisfação pessoal em referência à persistência dos estudantes dentro do programa acadêmico. Essas duas variáveis, permanência e persistência, são caracterizadas neste trabalho como processos independentes, suscetíveis ao estudo, que interagem e têm impacto direto em permanecer no ensino superior.

Palavras-chave: Abandono; permanência; ensino superior; educação técnica, evasão escolar.

\title{
Introducción
}

El estudio presentado parte de la búsqueda en todo el sistema educativo de procurar el éxito y bienestar estudiantil, basado en el presupuesto fundamental: conseguir que el estudiantado que ingresa a las aulas para cursar estudios en cualquier nivel de educación, lo culmine y se gradúe oportunamente.

En este proceso surgen determinados fenómenos objeto de análisis, tales como la deserción, la persistencia y la permanencia estudiantil; estos evidencian manifestaciones de que algo en el sistema educativo no responde a ciertas necesidades, y lleva al estudiantado a abandonar sus programas académicos. En este trabajo, nos fijamos en el nivel de educación superior (ES) en Colombia.

El término deserción ha sido ampliamente revisado por diferentes autores (Páramo y Correa, 1999; Tinto, 2010; Torrado, 2012, entre otros) para referirse a la representación estadística del porcentaje de estudiantes que abandonan las aulas de una institución de ES dada. En el caso particular de Colombia, la lectura de esta situación nos permite observar un alto porcentaje de abandono en la formación vinculada a ES, como puede apreciarse en la Tabla 1. 
Tabla 1: Deserción en los niveles de educación superior en Colombia. Año 2015

\begin{tabular}{lc}
\hline \multicolumn{1}{c}{ Nivel de formación } & Porcentaje abandono \\
\hline Técnica & $56,9 \%$ \\
Tecnológica & $52,1 \%$ \\
Universitaria & $46,1 \%$ \\
\hline
\end{tabular}

Nota: Adaptado diagrana SPADIES (MEN, 2015).

La reflexión sobre esta problemática constituye un reto para el sistema de ES desde comienzos del siglo XXI, debido a las exigencias de la sociedad globalizada y del mercado laboral.

El Ministerio de Educación Nacional (MEN) colombiano apoya las intervenciones institucionales que propendan por disminuir las cifras de deserción estudiantil en ES. El Sistema de Prevención de la Deserción en Educación Superior (SPADIES), creado por el MEN, posibilita que cada institución identifique y clasifique al estudiantado en riesgo de deserción mediante grupos determinados de variables. Con esta clasificación, el SPADIES busca determinar el efecto de las variables más significativas en los niveles de deserción dentro del agregado nacional, ofreciendo herramientas útiles a las instituciones de educación superior (IES) para el análisis y la prevención del fenómeno en cada contexto particular.

La necesidad de transcender de la representación y caracterización estadística del problema a la toma de decisiones es lo que impulsa a las IES a estudiar la realidad concreta de los fenómenos asociados al proceso de deserción, para comprender, así, sus dinámicas y buscar alternativas de solución para cada problemática concreta. En este sentido, nos fijaremos en una IES, como es el caso del Politécnico Internacional (PI) de Bogotá.

Al respecto, se formulan tres interrogantes sobre los factores asociados con la permanencia del estudiantado en las IES: ¿la persistencia se relaciona con las características del estudiantado, sus expectativas, intereses o situaciones particulares?; ¿la retención se relaciona con las dinámicas que se establecen entre la satisfacción de necesidades del estudiantado y la institución?; y, ¿la relación de las necesidades del estudiantado con las dinámicas institucionales se enlaza y asocia con la permanencia estudiantil?

Ante estas cuestiones nos proponemos los siguientes objetivos:

1. Identificar los factores que inciden en la retención estudiantil en el caso del Politécnico Internacional.

2. Describir las características relacionadas con la persistencia en el estudiantado del Politécnico Internacional.

3. Analizar los factores que inciden en la permanencia estudiantil, centrados en el estudiantado y en la institución. 
http://doi.org/10.15359/ree.25-1.6

http://www.una.ac.cr/educare

educare@una.ac.cr

\section{Apartado conceptual}

\section{Sobre la deserción estudiantil y la permanencia}

La deserción, desde la mirada de Tinto (2010), está relacionada directamente con las situaciones que enfrenta el estudiantado al no lograr culminar sus proyectos educativos. Páramo y Correa (1999) analizan el término, enunciando además las serias repercusiones sociales que conlleva el abandono de la formación académica estudiantil. Del mismo modo, se define deserción como "[el] abandono prematuro de un programa de estudios antes de alcanzar el título o grado ... [que] considera un tiempo suficientemente largo como para descartar la posibilidad de que el estudiante se reincorpore" (Himmel, 2002, pp. 94-95).

El fenómeno de la deserción estudiantil ha sido abordado desde el enfoque cuantitativo y cualitativo. El primero, al ofrecer una visión a gran escala de las tendencias y porcentajes de deserción, ha sido utilizado en la mayoría de los casos. Se concibe al estudiantado como unidad de análisis para la obtención de cifras que develen causas y factores de riesgo, con el objetivo de generar relaciones que permitan estudiarlo en profundidad según cada contexto (Barrero, 2015).

Por otra parte, el enfoque cualitativo, aunque utilizado en menor medida, ha ofrecido una lectura del fenómeno del abandono asociándolo a problemas culturales en la educación, otorgando significaciones individuales y sociales a los facilitadores de riesgo para no permanecer en el sistema educativo (Barrero, 2015).

De igual manera, diversos modelos de descripción o explicación de la deserción han de tenerse presentes, en la medida en que facilitan la comprensión y análisis del fenómeno. Himmel (2002) describe los siguientes:

- El modelo de deserción de Spady, que combina factores básicos para el entendimiento y la exploración de las variables que afectan al estudiantado e inciden en la toma de la decisión de desertar. Los antecedentes familiares encabezan este modelo como el factor de donde parte la motivación individual para estudiar y continuar con el programa elegido hasta culminar.

- El modelo de Tinto, soportado sobre tres bases: antecedentes familiares, personales y preuniversitarios. Factores que se interrelacionan para repercutir directa o indirectamente en la decisión del estudiantado de continuar o desertar del programa en el que se matriculó.

- El modelo de Bean, que complementa las bases del modelo de Tinto con los factores ambientales y la dimensión socialización del estudiantado en su entorno próximo. Elementos que pueden o no conducir a la deserción. 
http://doi.org/10.15359/ree.25-1.6

Dentro de los modelos enunciados, uno de los más influyentes ha sido el trabajado por Tinto, 1993 (citado en Báez et al., 2010), quien en sus planteamientos reconoce que para hablar de permanencia se deben abarcar no solo los antecedentes familiares, personales y académicos del estudiantado, sino también las condiciones de integración. Estas dimensiones se desarrollan entre la institución y el estudiantado. Las instituciones se involucran en tanto constituyen la base estructural para el desarrollo de potenciales del estudiantado desde el ingreso hasta la culminación de su proceso académico (Tinto, 1989).

Se anotan, además, los factores psicológicos (actitudes, motivaciones, autoeficacia, atribución de causa) como rasgos relevantes e influyentes para la continuidad o el abandono de los estudios (Apaza y Huamán, 2012). Por tanto, se establece, en las instituciones, la utilización de diversas herramientas de bienestar para el estudiantado, como soporte y factor motivacional para continuar asistiendo a la universidad.

\section{Factores de riesgo y protección asociados a la retención}

Es útil definir los factores de riesgo que han sido asociados de manera significativa con la deserción, en la medida en que permitirán facilitar el análisis de las relaciones causales y orientar acciones futuras. Apuntamos los siguientes:

1. Antecedentes familiares y culturales: los niveles educativos de los padres y madres $y$, en general, los niveles académicos alcanzados dentro del entorno familiar del estudiantado, influyen directamente sobre las decisiones educativas que tomará durante su vida. Deben asociarse los factores culturales que forman parte del contexto del estudiantado, ya que influyen en sus creencias, expectativas o costrumbres sociales (González-Ramírez y Pedraza-Navarro, 2017; Munizaga et al., 2018).

2. Atributos personales o individuales: la motivación personal, el interés por progresar y mejorar la calidad de vida y la percepción individual acerca de las actividades académicas que se llevarán a cabo al ingresar a la universidad influyen directamente en la decisión de inscribirse a cursar estudios de nivel superior, así como de continuar y culminar con la obtención del título (Himmel, 2002; Munizaga et al., 2018).

3. Escolarización preuniversitaria: el nivel de escolaridad previo al ingreso a la ES influye sobre la decisión del estudiantado en la elección del programa que desea cursar (Torres Guevara (2012).

4. Factores académicos: hacen referencia a los aspectos intelectuales, de desempeño y de rendimiento académico con los que el estudiantado cuenta para avanzar en su proceso académico (Tuero et al., 2018), en su trayectoria académica (Munizaga et al., 2018). Mediante la integración de estos factores académicos, el individuo comprende, 
http://doi.org/10.15359/ree.25-1.6

http://www.una.ac.cr/educare

educare@una.ac.cr

interioriza y proyecta el desempeño que debe tener para lograr obtener el título y culminar con éxito su proceso académico (Himmel, 2002).

5. Factores psicológicos: el estudiantado, como ser integral, debe ser tenido en cuenta y valorado en todas sus dimensiones para generar en él la motivación necesaria que le permita continuar en su proceso de formación. No debe desligarse el componente psicológico del individuo, o anteponer el componente académico por encima del ser, ya que se incurre en un desligamiento del ser con el saber, lo cual promueve la insatisfacción personal que genera la posibilidad de abandonar los estudios (Torres Guevara, 2012).

6. Factores ambientales: muy considerados en sociedades con bajos recursos, en donde se suman, además de la motivación, los antecedentes personales y los factores académicos e institucionales, entre otros (Himmel, 2002).

7. Factores sociales: la exclusión social, entendida como el quebrantamiento de los vínculos sociales, tarde o temprano desligará al estudiantado de sus estudios, bien sea por la situación económica o por la situación individual o social de su entorno, aspecto común de la ruptura de lazos en la relación individuo - sociedad (Torres Guevara, 2012).

8. Factores económicos: causas socioeconómicas fundamentadas en la incompatibilidad laboral y en los recursos de acceso a la financiación (íñiguez et al., 2016; Munizaga et al., 2018).

La relevancia de la implementación de prácticas enfocadas en la permanencia y la retención estudiantil,-basadas en los contextos particulares de cada universidad, debe tenerse en cuenta, para obtener resultados eficaces (Korantowicz y Nizinska, 2013). Para tal fin, cada institución atenderá variables y categorías orientadas al estudio de su situación particular, partiendo de las formuladas como estándares del sistema educativo. En el presente contexto, el MEN (2009) establece: variables individuales (edad, género, estado civil, calamidad y problemas de salud, incompatibilidad horaria con actividades extra académicas, expectativas no satisfechas, embarazo); académicas (orientación profesional, calidad del programa, insatisfacción con el programa); institucionales (becas y formas de financiamiento, recursos universitarios, nivel de interacción personal con docentes y estudiantes, apoyo académico, apoyo psicológico); socioeconómicas (estrato, situación laboral, situación laboral de las madre y padres e ingresos, dependencia económica, personas a cargo, nivel educativo de la familia).

Para el MEN (2015) "la permanencia se entiende como un tema ocasionado por múltiples causas en el que intervienen diferentes actores y que más allá de un asunto económico, ... tiene también un impacto directo en el proyecto de vida del estudiante y su familia" (p. 19). Meléndez Surmay y Meriño Mendoza (2008) la definen como el tiempo que tarda cada estudiante en terminar los requisitos académicos para obtener el título profesional. También es definida 
http://doi.org/10.15359/ree.25-1.6

como el proceso vivido por el estudiantado desde el ingreso, el transcurso del programa y su culminación en un tiempo determinado, teniendo en cuenta factores adicionales como la obtención de promedios ponderados adecuados, el establecimiento de relaciones sociales, la formación previa del estudiantado, su historia académica, su situación socioeconómica, la capacidad de adaptación y la resistencia y la tolerancia a la frustración (Velásquez et al., 2011).

\section{Sobre la permanencia- deserción en educación superior}

Las investigaciones relacionadas con el abandono estudiantil son fuente de recursos para emprender la transición y evolución desde el fenómeno de deserción hacia el de permanencia. Términos como mortalidad estudiantil, deserción universitaria, deserción estudiantil, retención, persistencia y permanencia (Fernández-Hileman et al., 2014) se han utilizado con frecuencia al discutir y profundizar sobre el tema, obviando las características y matices diferentes que los hacen conceptos divergentes en la realidad.

En relación con la deserción, la revisión documental se centra en el examen de los perfiles del estudiantado que abandona los estudios, teniendo en cuenta para ello, entre otros, factores personales, económicos, sociales e institucionales (Munizaga et al., 2018). El término permanencia, por el contrario, contiene una cantidad significativa de variables y características, que deben ser revisadas y analizadas por las instituciones y los sistemas de educación, con el fin de comprender y mitigar el abandono estudiantil.

La relación permanencia-deserción involucra dimensiones anexas, tales como la persistencia y la retención estudiantil. Estas dimensiones forman parte de la explicación y profundizan en el fenómeno.

Persistencia es la capacidad del estudiantado, en los niveles académico, social y motivacional, de permanecer en la institución hasta la obtención del título; es la búsqueda por alcanzar sus propias metas educativas. Es una medida centrada en el estudiantado y, por lo tanto, representa su punto de vista (Torres Guevara, 2012).

La retención, como dimensión asociada a la permanencia estudiantil, hace referencia al rol de las instituciones y a su capacidad por mantener matriculado al estudiantado desde el ingreso hasta la graduación. Representa la perspectiva institucional, desde la cual se realizan y aplican estrategias según el contexto particular, para lograr la permanencia del estudiantado hasta su graduación (Torres Guevara, 2012).

De manera análoga, la retención está ligada a las acciones institucionales que garanticen el acompañamiento del estudiantado durante su proceso académico; acciones que deben asegurar la culminación exitosa del programa académico, siempre en el marco del desarrollo de las competencias, aptitudes y conocimientos necesarios y pertinentes para el futuro laboral (Pineda-Báez et al., 2011). 
http://doi.org/10.15359/ree.25-1.6

http://www.una.ac.cr/educare

educare@una.ac.cr

\section{Apartado metodológico}

El presente estudio se centra en el caso del Politécnico Internacional (P.I) Bogotá, Colombia, por lo que se corresponde con el estudio de una institución, donde se valoran evidencias que permiten la triangulación y proposiciones teóricas que guían el análisis de los datos (Stake, 2007; Yin, 2009); pertenece a los denominados como ex post facto, ya que procura investigar las posibles relaciones de causa-efecto (Cohen et al., 2007), comparando las semejanzas y diferencias que existen entre fenómenos, para descubrir los factores que contribuyen a la aparición de situaciones (Rosado, 2006).

El estudio observa la condición de permanencia estudiantil institucional, buscando respuestas plausibles como factores causales; es decir, qué factores están asociados a la permanencia. De igual manera, está encaminado a visibilizar las relaciones entre diferentes variables (Rosado, 2006) dentro de la estructura del PI.

Este estudio de caso "trata de explicar el carácter evolutivo y complejo de los fenómenos que conciernen a un sistema social que tiene sus propias dinámicas" (Mucchielli, 2001, p. 102) y tiene como objetivo principal contextualizar en la vida real un fenómeno específico, teniendo en cuenta diferentes fuentes de información. Por tal razón se toma el evento tal como ocurre en forma natural en la institución y se estudia, sin controlarlo.

La recolección de la información, dentro del contexto en que fue obtenida, se fundamentó en la visión del estudiantado para comprender la relevancia de los factores que influyen en la permanencia hasta la culminación de los estudios y el recibimiento del grado en el alumnado de la institución.

\section{Población y muestra}

La selección de estudiantes del PI se realizó teniendo en cuenta los siguientes parámetros:

a. Contar con matrícula vigente período académico 2017-1 en alguno de los programas académicos de la institución, en todos los niveles de primero a décimo, incluyendo el programa de inglés.

b. Pertenecer a la sede principal de la institución. Existen tres sedes adicionales en la ciudad de Bogotá (Kennedy, Sur y Calle 80).

c. Estar matriculado en cualquier jornada ofrecida por la institución (diurna, especial, tarde, nocturna).

d. Asistir en las semanas de clase 6 y 7 del calendario académico. 
http://doi.org/10.15359/ree.25-1.6

e. Responder la evaluación institucional posterior al diligenciamiento del cuestionario de permanencia estudiantil (ingreso a la página web de la institución y acceder al link que lo dirigía primero a contestar el formulario de permanencia y una vez finalizado este, a continuar con la evaluación docente estudiantil).

La muestra obtenida es de 1 487, correspondiente a una población de 3411 estudiantes con matrícula para el período académico 2017-1, momento en el cual fueron aplicados los instrumentos; con un margen de error del 2,5\% y un nivel de confianza del 99\%, se utilizó la ecuación estadística para proporciones poblacionales. Hay que especificar que el presente trabajo forma parte de uno más amplio y se centra, en esta ocasión, en la perspectiva del estudiantado.

\section{Instrumentalización}

Los instrumentos para la recolección de la información utilizados en la presente investigación han sido cuestionarios ad-hoc. La elaboración de los cuestionarios se realizó con base en modelos de variables tomadas de revisiones bibliográficas, cifras del MEN e información preliminar de la institución, entre otras, se tuvieron en cuenta los diferentes determinantes que se exponen en la Tabla 2, los cuales han sido cimiento para la obtención de resultados en el análisis del fracaso escolar y del abandono.

Los cuestionarios fueron validados por jueces, que analizaron el contenido y el tipo de respuesta en función de los objetivos de la investigación, desde los criterios de univocidad, pertinencia e importancia; lo que dio como resultado, a partir de las modificaciones sugeridas por los validadores, las versiones definitivas de los cuestionarios.

Tabla 2: Estructuración de los determinantes de la deserción estudiantil para la conformación de los cuestionarios

\begin{tabular}{llll}
\hline \multicolumn{1}{c}{ Determinantes individuales } & \multicolumn{1}{c}{$\begin{array}{c}\text { Determinantes } \\
\text { académicas }\end{array}$} & $\begin{array}{c}\text { Determinantes } \\
\text { institucional }\end{array}$ & $\begin{array}{c}\text { Determinantes } \\
\text { socioeconómicos }\end{array}$ \\
\hline $\begin{array}{l}\text { Edad, género, estado civil } \\
\begin{array}{l}\text { Posición dentro de los } \\
\text { hermanos/as }\end{array}\end{array}$ & $\begin{array}{l}\text { Orientación profesional } \\
\text { Rendimiento académico }\end{array}$ & Programas de bienestar & Estrato \\
Entorno familiar & Calidad del programa & Recursos universitarios & Ingresos familiares \\
Problemas de salud, embarazo & $\begin{array}{l}\text { Insatisfacción con el } \\
\text { programa }\end{array}$ & Apoyo académico & Personas a cargo \\
Horarios laborales & & Apoyo psicológico & Nivel educativo de los \\
& & & padres y madres \\
Expectativas no satisfechas & & & Medio de transporte \\
\hline
\end{tabular}

Nota: Adaptado de diagrama 4 (MEN, 2009). 
http://doi.org/10.15359/ree.25-1.6

http://www.una.ac.cr/educare

educare@una.ac.cr

Con estas características, los cuestionarios definitivos estuvieron compuestos por 31 ítems Algunos ítems del cuestionario que se refieren a los motivos de asistencia y de abandono se han conformado como dimensiones, al disponer de subítems o elementos (33 y 34), sometidos al análisis de su fiabilidad utilizando el coeficiente $\alpha$ de Cronbach, cuyo resultado para el ítem 33 (14 elementos) el 0,956 y para el ítem 34 (22 elementos) el 0,953; se observa, en este sentido, una fuerte consistencia interna. Se ha procedido, en este caso, a la realización de un análisis factorial exploratorio (AFE), KMO, mediante el estudio de los componentes principales y el método de rotación Varimax con normalización Kaiser, que confirma dos factores para los elementos del ítem 33, explicando el 77,7\% de la varianza; y para los elementos del ítem 34 dos factores, que explican el $58 \%$ de la varianza.

Tabla 3: Relación ítem-factor motivos asistencia

\begin{tabular}{|c|c|c|}
\hline & \multicolumn{2}{|c|}{ Factor } \\
\hline & MOT_Asiste1 & MOT_Asiste2 \\
\hline 1. Asistencia a clases pensando en su futuro laboral & $x$ & \\
\hline 2. Asistencia a clases por sus compañeros & & $\mathrm{X}$ \\
\hline 3. Asistencia a clase por las instalaciones & & $\mathrm{X}$ \\
\hline 4. Asistencia a clase por compromiso adquirido & $\mathrm{X}$ & \\
\hline 5. Asistencia a clases por interés laboral & $x$ & \\
\hline 6. Asistencia a clases por aprender & $x$ & \\
\hline 7. Asistencia a clases por el personal docente & & $X$ \\
\hline 8. Asistencia a clases por la metodología del personal docente & $x$ & $\mathrm{X}$ \\
\hline 9. Asistencia a clases para mejorar la calidad de vida & $X$ & \\
\hline 10. Asistencia a clases por el apoyo académico & $x$ & $\mathrm{X}$ \\
\hline 11. Asistencia a clases por calidad del programa & $X$ & $X$ \\
\hline 12. Asistencia a clases por adquirir conocimientos & $X$ & \\
\hline 13. Asistencia a clases por formación profesional & $\mathrm{X}$ & \\
\hline 14. Asistencia a clases para preparación laboral & $\mathrm{X}$ & \\
\hline
\end{tabular}

Nota: Elaboración propia.

Los motivos de asistencia a clases tenidos en cuenta por el estudiantado han sido destacados en la Tabla 3. El Factor MOT_Asiste1, se relaciona con características asociadas al futuro y preparación laboral, presenta indicadores tales como compromiso, interés laboral, calidad del programa o adquisición de conocimientos . 
El Factor MOT_Asiste2 se relaciona con características asociadas a los recursos materiales y humanos, a la metodología docente y al apoyo académico.

Tabla 4: Relación ítem-factor motivos abandono

\begin{tabular}{llc}
\hline & \multicolumn{2}{c}{ Factor } \\
\cline { 2 - 3 } & MOT_Abandona1 & MOT_Abandona2 \\
\hline 1. Aplazaría por motivos económicos & & $\mathrm{X}$ \\
\hline 2. Aplazaría por falta de tiempo & & $\mathrm{X}$ \\
\hline 3. Aplazaría por situación laboral & $\mathrm{X}$ \\
\hline 4. Aplazaría por dificultades familiares & $\mathrm{X}$ \\
\hline 5. Aplazaría por problemas de salud & $\mathrm{X}$ \\
\hline 6. Aplazaría por embarazo & $\mathrm{X}$ \\
\hline 7. Aplazaría por espectativas no satisfechas & $\mathrm{X}$ \\
\hline 8. Aplazaría por conflicto con docente & $\mathrm{X}$ \\
\hline 9. Aplazaría por convivencia con compañeros/as & $\mathrm{X}$ \\
\hline 10. Aplazaía por bajo rendimiento académico & $\mathrm{X}$ \\
\hline 11. Aplazaría por cambio de ciudad o pais & $\mathrm{X}$ \\
\hline 12. Aplazaría por viaje temporal & $\mathrm{X}$ \\
\hline 13. Aplazaría por cambio de institución & $\mathrm{X}$ \\
\hline 14. Aplazaría por calidad del programa & $\mathrm{X}$ \\
\hline 15. Aplazaría por insatisfacción con la institucion & $\mathrm{X}$ \\
\hline 16. Aplazaría por repetir materias & $\mathrm{X}$ \\
\hline 17. Aplazaría por apoyo financiero & $\mathrm{X}$ \\
\hline 18. Aplazaría por el perfil del programa & $\mathrm{X}$ \\
\hline 19. Aplazaría por cambio de perfil profesional & $\mathrm{X}$ \\
\hline 20. Aplazaría por metodología enseñanza/aprendizaje & $\mathrm{X}$ \\
\hline 21. Aplazaría por no responder exigencias académicas & $\mathrm{X}$ \\
\hline 22. Aplazaría por cambio de perspectiva & \\
\hline
\end{tabular}

Nota: Elaboración propia. 
http://doi.org/10.15359/ree.25-1.6

http://www.una.ac.cr/educare

educare@una.ac.cr

De igual manera, los motivos por los cuales el estudiantado refirió que llegaría a abandonar sus estudios, han sido destacados en la Tabla 4. MOT_Abandona1, se relacionan con características asociadas con dificultades familiares, individuales, institucionales, académicas y financieras

MOT_Abandona 2, se relaciona con características asociadas con dificultades económicas, principalmente, seguidas por familiares y de salud.

\section{Procedimiento}

Los cuestionarios validados en su versión final se transcribieron utilizando la herramienta para encuestas Google Drive. Se realizaron pruebas de los formularios vía web, para contar con la seguridad del correcto funcionamiento. Posteriormente fueron aplicados durante las semanas seis y siete del primer período académico del año 2017 (2017-1T) en los meses de febrero y marzo al estudiantado de las cuatro jornadas y que contaba con las demás características señaladas anteriormente. Durante el primer día de aplicación de los cuestionarios, surgieron observaciones importantes en dos preguntas, las cuales fueron corregidas según criterios y ajustes pertinentes para minimizar la invalidación de preguntas durante la fase de análisis de la información. Teniendo en cuenta que durante cada período académico en el PI se realizan valoraciones masivas, bien sea a nivel de evaluación docente o institucional, en la página web corporativa, se optimizó la aplicación del cuestionario de permanencia estudiantil ligando el link a la evaluación docente con dos fines principales:

1. Obtener la mayor cantidad posible de cuestionarios respondidos.

2. Evitar agotar al estudiantado contestando durante el mismo período académico dos cuestionarios con información institucional.

La información para el correcto diligenciamiento del cuestionario de permanencia estudiantil fue transmitida por medio de los decanatos de las facultades al personal docente de las asignaturas, con el fin que guiaran al estudiantado al momento de aplicarlo, resolviendo inquietudes que se pudieran presentar. Se aseguró el consentimiento informado del estudiantado en el cual se les garantiza la confidencialidad. Una vez cada estudiante accedía al link, el primer cuestionario que aparecía en la página era el de permanencia estudiantil, el que debía ser diligenciado en su totalidad, para poder acceder al de evaluación docente. Finalizadas las semanas para el diligenciamiento del cuestionario, se consolidó la información y se obtuvieron los datos definitivos mediante el programa SPSS, con los que nos permitimos realizar el análisis descriptivo e inferencial, dando cumplimiento a los objetivos específicos del estudio. 
http://doi.org/10.15359/ree.25-1.6 http://www.una.ac.cr/educare educare@una.ac.cr

\section{Resultados}

La relación de las dimensiones tomadas para el análisis de las variables que inciden en la permanencia estudiantil cimentan los resultados de la presente investigación (Figura 1). Previo al inicio de la identificación de las dimensiones en mención, se describe, inicialmente, la caracterización de la muestra, para dar claridad y organización a los resultados.

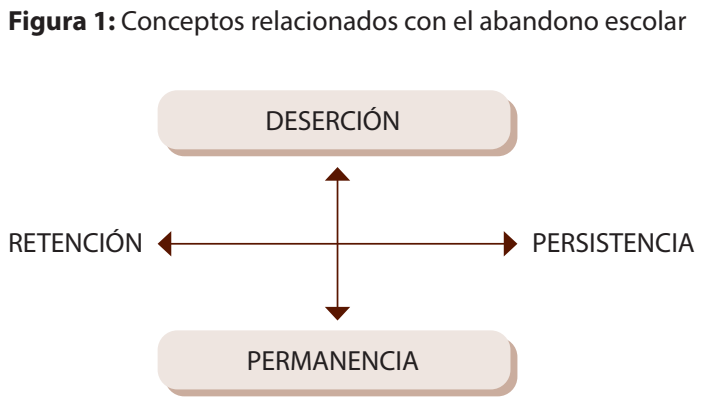

Nota: Elaboración propia.

\section{Caracterización de la muestra de estudiantes}

Las variables edad, género, estado civil y estado socioeconómico fueron tomadas para estructurar la información y perfilar los resultados teniendo en cuenta dichos parámetros. Con el fin de relacionar los factores bajo medidas estandarizadas, se tomaron los grupos de mayor representación de cada variable de la muestra para la descripción y análisis de los hallazgos, tal como se expone a continuación:

1. Edad. El mayor porcentaje de la muestra (39,9\%), se ubica en el rango de 18 a 20 años. Seguidos por el grupo comprendido entre los 21 a 24 años de edad con un 29,3\% de participación. El estudiantado menor de edad $(<18)$ representan el menor porcentaje de la muestra $(13,4 \%)$.

2. Género. Las mujeres ocupan el mayor porcentaje en la muestra de estudiantes encuestados (55,5\%). Los hombres el 42,6\%, y el estudiantado que prefiere no decir su género el $1,8 \%$.

3. Estado civil. La mayoría del estudiantado de la muestra es soltero (84,1\%). Distantes de esta población, en segundo lugar se encuentra el estudiantado que vive en unión libre con sus parejas (8,5\%), seguido por el estudiantado casado (3,6\%).

4. Estrato socioeconómico. El mayor porcentaje de estudiantes de la muestra pertenece al estrato socioeconómico 3 (nivel socioeconómico medio en Colombia) (43,6\%). En segundo lugar se encuentra el estudiantado del estrato 2 (nivel socioeconómico bajo 
en Colombia) (35,8\%). Los estratos socioeconómicos con menor representación en la muestra son el 1 (9\%), el 4 (8,3\%), el 5 (1,9\%) y el 6 (nivel socioeconómico alto en Colombia) con un 1,3\% de participación.

\section{Identificación de factores de retención estudiantil en el PI}

Se ha incidido sobre los siguientes factores de retención: aplazamiento de estudios por cambio de institución, por calidad del programa, por apoyo financiero y por perfil del programa.

En función de la variable edad del estudiantado, cabe destacar, en general, que los valores de las medias se sitúan por debajo de la media teórica $(=2,5)$, exceptuando "mayor de 25 años" ( $m e d i a=2,54)$ en relación con la calidad del programa como factor relevante. Esto nos hace pensar en el bajo nivel de incidencia de los factores establecidos asociados a la retención desde la variable edad del estudiantado.

De igual manera que la variable edad, la variable género no parece incidir considerablemente en los motivos conformados como factores de retención del estudiantado en la continuación de los estudios, ya que todas las medias se encuentran por debajo de la media teórica.

Respecto el estado civil, hay que destacar que los viudos muestran medias superiores a la teórica en el aplazamiento por cambio de institución (media= 2,68), por calidad del programa (media $=2,66$ ) y por apoyo financiero (media $=2,54$ ); así como la categoría de persona divorciada, en aplazamiento por cambio de institución (media=2,63) y por perfil del programa (media $=2,71$ ). En el resto de categorias, no resulta considerable el nivel de incidencia de los factores establecidos asociados a la retención.

Asimismo, los valores de las medias basados en los estratos socioeconómicos se sitúan, en su mayoría, por debajo de la media teórica $(=2,5)$, exceptuando el estrato 5 para las cuatro variables consideradas, donde se observan resultados por encima de la media teórica (cambio de institución=2,84, calidad del programa=2,79, apoyo financiero=2,64 y perfil del programa=2,92), respectivamente.

Por otra parte, el análisis ANOVA permite establer las diferencias entre las distintas variables especificadas; concretamente, en relación con la calidad de la institución PI, pueden observarse diferencias significativas, en función de la variable género $(<0,05)$ entre quienes prefieren no mencionar su género y quienes sí lo hacen (hombres y mujeres), y se determina una menor puntuación por parte del primer grupo. Asimismo, la variabe estrato social aparece como influyente en indicadores establecidos para la toma de decisión sobre el ingreso en la institución (calidad, facilidades de pago, recomendación de una amistad o familiar, horarios, bienestar y ubicación), atendiendo que el estrato 5 puntúa significativamente por debajo de los demás estratos sociales $(<0,05)$. 
http://doi.org/10.15359/ree.25-1.6

\section{Características asociadas a la persistencia en el PI}

En cuanto al aplazamiento de estudios como variable asociada a la persistencia estudiantil, se incidió, como puede observarse en la Tabla 5, en tres factores destacados por los cuales el estudiantado llegaría a aplazar sus estudios en el PI, teniendo en cuenta su puntuación por encima de la media teórica $(=2,5)$. Estos son: los motivos económicos $(2,96)$, la situación laboral $(2,51)$, y los cambios de ciudad o país $(2,53)$.

Tabla 5: Factores asociados a la persistencia estudiantil en el Politécnico Internacional

\begin{tabular}{llr}
\hline Variable & Media & DS \\
\hline Aplazar por motivos económicos & 2,96 & 1,059 \\
Aplazar por situación laboral & 2,51 & 1,067 \\
Aplazar por cambio de ciudad o país & 2,53 & 1,171 \\
\hline
\end{tabular}

Nota: Elaboración propia.

De esta forma, se corrobora que tanto los motivos económicos como los laborales inciden directamente y en mayor medida sobre la toma de decisón del estudiantado para abandonar sus estudios; información coincidente con los estudios de diagnóstico y seguimiento de la permanencia estudiantil realizados en el país por el Ministerio de Educación Nacional MEN (2009).

\section{Análisis de los factores familiares que inciden en la permanencia en el PI}

El análisis de la permanencia estudiantil ha sido efectuado con la adaptación en contexto de uno de los modelos de mayor influencia desarrollado por Tinto (1993, citado en Báez et al. 2010). Por ello, se tiene en cuenta los incorporados a las variables familiares y sus relaciones.

\section{Antecedentes familiares relacionados con estudio y trabajo}

Escolaridad del padre: según el estudiantado encuestado el 27,9\% de sus padres ha alcanzado el nivel de estudios de ES. En educación técnica y tecnológica el 11,63\%; en nivel universitario completo el 9,41\%; en nivel universitario incompleto el 3,63\%; y con estudios de posgrado el 3,3\%. Básica secundaria un 32,48\% y básica primaria el 21,79\%. Por otro lado, en un $1,75 \%$ el padre nunca estudió y el estudiante no sabe si su padre estudió en un 16,01\%. Podemos así concluir que el nivel educativo alcanzado por los padres del estudiantado, en mayor porcentaje, es el nivel básico, con un total entre básica primaria y secundaria de 54,7\%. 
http://doi.org/10.15359/ree.25-1.6

http://www.una.ac.cr/educare

educare@una.ac.cr

Escolaridad de la madre: con la escolaridad de la madre ocurre algo similar a lo encontrado con la escolaridad paterna, un 34,05\% llegó a nivel de estudios superiores, con educación técnica y tecnológica el 15,47\%, nivel universitario completo el 10,37\%, nivel universitario incompleto 4,84\% y con estudios de posgrado solamente el 3,83\%. En básica secundaria 37,19\%; concluida el $24,95 \%$ y no concluida el $12,24 \%$. En básica primaria el $20,38 \%$; incompleta el $10,42 \%$ y completa el 9,95\%.

El porcentaje de madres que no estudiaron es del 1,48\% y el 6,46\% restante corresponde a los datos de estudiantes que no saben si sus madres estudiaron.

Escolaridad de los hermanos o hermanas: en el caso de los hermanos o hermanas se observa que el $46,07 \%$ ha alcanzado un nivel de estudios en educación superior; con estudios de posgrado el 4,84\%, universitaria completa el 15,06\%, universitaria incompleta el 8,14\%, técnica y tecnológica el 18.02\%. A nivel de educación secundaria el 31,67\%, completa 23,81\% e incompleta el 7,87\%. A nivel de primaria el 8,88\%, completa 5,31\%, e incompleta 3,56\%. Además, sin estudios se encuentra un 2,15\% y no sabe el nivel de educación de sus hermanas o hermanos el 11,23\%. Los resultados muestran que los hermanos y hermanas del estudiantado de la institución, en más alto porcentaje, han alcanzado estudios de educación superior.

Ocupación del padre: los datos exponen que el más alto porcentaje de los padres se encuentran trabajando de manera independiente $(41,4 \%)$, seguidos por los que se encuentran empleados $(35,2 \%)$ y sin trabajo el $12,3 \%$.

Ocupación de la madre: el más alto porcentaje de las madres de estudiantes de la institución, se encuentran empleadas (41,5\%), seguidas por las que se encuentran trabajando de manera independiente (33\%).

Prioridades de la familia en cuanto a estudio: el estudiantado del PI considera que los estudios que lleva a cabo, ocupan una alta (47\%) o muy alta $(30,9 \%)$ prioridad dentro de su familia, una prioridad media el $17,7 \%$ y solo una prioridad baja y muy baja el $4,5 \%$.

\section{Características socio-familiares}

Número de hijos o hijas: la gran mayoría de estudiantes de la muestra poblacional de la institución (83,6\%) no tiene hijos ni hijas; el 10,2\% tienen 1 y el resto (4,7\%) 2 o 3.

Número de hermanos o hermanas: la mayoría de estudiantes (66\%) tienen 1 o 2 hermanos. Tres o más el $29,4 \%$ y ningún o ninguna el 4,6\%.

Ocupación laboral: el 51,71\% de estudiantes se encuentra trabajando, el 48,29\% no trabaja. De quienes trabajan, el 25,8\% tiene contrato a término indefinido, el 7,9\% a término definido, el $8,3 \%$, por prestación de servicios y el 15,9\% trabaja de manera informal. 
http://doi.org/10.15359/ree.25-1.6

Relación del trabajo con los estudios: el 43,8\% de estudiantes no trabaja en temas relacionados con sus programas de estudio en el PI. El 19,8\% sí tiene trabajos relacionados con sus programas de estudio.

Puede aludirse, por lo general, la prevalencia de antecedentes familiares paternos y maternos relacionados con el nivel cultural formal de básica primaria y secundaria y de estudios superiores y de educación secundaria en hermanos o hermanas que caracteriza el perfil del alumnado del PI. Asimismo, se observa un perfil de estudiantes dominado por la compaginación estudio-trabajo, como aspectos más sobresalientes.

\section{Análisis de factores motivacionales}

Para el análisis de factores motivacionles relacionados con la asistencia y abandono, hemos utilizado específicamente el análisis de varianza ANOVA, que nos permite determinar diferencias entre distintas categorías dentro de una misma variable, incluyendo prueba posthoc de Scheffe, por lo que podemos inferir su incidencia (ver Tabla 6). Se han tenido presente algunas de las variables apuntadas en los determinantes descritos en la Tabla 2.

Tabla 6: Análisis inferencial de factores motivacionales

\begin{tabular}{|c|c|c|c|c|}
\hline \multicolumn{5}{|c|}{ F ACTORES MOTIVACIONALES DE ASISTENCIA Y ABANDONO } \\
\hline VARIABLE & MOT_Asiste1 & MOT_Asiste2 & MOT_Abandona1 & Mot_Abandona2 \\
\hline Género & $\begin{array}{l}\text { Prefiere no decir }<\text { hombre, mujer } \\
(<.001)\end{array}$ & - & - & - \\
\hline Edad & - & - & $\begin{array}{l}<18 \text { a }<\text { todos/as los/as } \\
\text { demás } \\
(<.05)\end{array}$ & $\begin{array}{l}<18 \text { a. }<\text { todos/as los/as } \\
\text { demás } \\
(<.05)\end{array}$ \\
\hline $\begin{array}{l}\text { Estrato } \\
\text { socioeconómico }\end{array}$ & $\begin{array}{l}5<1,4,3,2 \\
6<3,2 \\
(<.001)\end{array}$ & $\begin{array}{l}5<2 \\
(<.05)\end{array}$ & $\begin{array}{l}5<6 \\
(<.05)\end{array}$ & $\begin{array}{l}5<3,6,2 \\
(<.05)\end{array}$ \\
\hline Estado Civil & $\begin{array}{l}\text { Divorciado/a < casado/a, m-p } \\
\text { soltero/a, unión libre,soltero/a; } \\
\text { Viudo/a }<\mathrm{m}-\mathrm{p} \text { soltero, unión } \\
\text { libre,soltero } \\
(<.001)\end{array}$ & $\begin{array}{l}\text { Divorciado/a < casado/a, } \\
\text { m-p soltero/a, unión } \\
\text { libre,soltero/a } \\
(<.001)\end{array}$ & - & - \\
\hline $\begin{array}{l}\text { Fuente de } \\
\text { ingreso }\end{array}$ & - & - & - & $\begin{array}{l}\text { Beca-Subsidio < Crto } \\
\text { educativo } \\
(<.05)\end{array}$ \\
\hline Realizar trabajo & - & - & - & $\begin{array}{l}\text { No }<\text { Sí } \\
(<.05)\end{array}$ \\
\hline
\end{tabular}


http://doi.org/10.15359/ree.25-1.6

http://www.una.ac.cr/educare

educare@una.ac.cr

\begin{tabular}{|c|c|c|c|c|}
\hline \multicolumn{5}{|c|}{ F ACTORES MOTIVACIONALES DE ASISTENCIA Y ABANDONO } \\
\hline VARIABLE & MOT_Asiste1 & MOT_Asiste2 & MOT_Abandona1 & Mot_Abandona2 \\
\hline $\begin{array}{l}\text { Ingresos } \\
\text { familiares }\end{array}$ & - & - & - & $\begin{array}{l}\text { Padres o madres acudientes } \\
<\text { recursos propios } \\
(<.05)\end{array}$ \\
\hline Ciclo académico & $\begin{array}{l}10<1 \\
(<001)\end{array}$ & $\begin{array}{l}6<1 \\
(<001)\end{array}$ & $\begin{array}{l}1<2,5,7,8 \\
(<.05)\end{array}$ & $\begin{array}{l}1<5 \\
(<.05)\end{array}$ \\
\hline Jornada estudio & $\begin{array}{l}\text { Tarde }<\text { diurno } \\
(<.05)\end{array}$ & & $\begin{array}{l}\text { Tarde }<\text { nocturno, } \\
\text { especial } \\
(<.05)\end{array}$ & $\begin{array}{l}\text { Tarde }<\text { Nocturno, diurno } \\
\text { Especial }<\text { Nocturno } \\
(<.05)\end{array}$ \\
\hline $\begin{array}{l}\text { Exigencia } \\
\text { Académica }\end{array}$ & $\begin{array}{l}\text { Muy bajo < Bajo < Muy alto } \\
(<.05)\end{array}$ & $\begin{array}{l}\text { Bajo, Muy bajo }<\text { Muy alto } \\
(<.05)\end{array}$ & & \\
\hline $\begin{array}{l}\text { Calificación } \\
\text { docente }\end{array}$ & $\begin{array}{l}\text { Regular,Mal< Bien,Muy bien, } \\
\text { Excelente } \\
(<.05)\end{array}$ & $\begin{array}{l}\text { Regular }<\text { Mal, Muy Bien, } \\
\text { Excelente }(<.05) \\
\text { Bien }<\text { Excelente } \\
(<.05)\end{array}$ & & \\
\hline
\end{tabular}

Nota: Elaboración propia.

Como podemos observar en la Tabla 6, los motivos de asistencia definidos como "MOT_ Asiste1", asociados al futuro y preparación laboral, vienen afectados por variables tales como el género, en aquellos casos que prefieren no definirse que se diferencian significativamente de los que responden como hombre o mujer; el estrato socioeconómico, entendiendo que encontramos diferencias significativas (<.001) entre el 5 y los estratos 1, 2, 3 y 4 . Asimismo, en relación con el estado civil, encontramos diferencias entre personas divorciadas y las casadas, solteras y en unión libre (<.001) y entre los sujetos viudos y madre-padre solteros, unión libre, solteros (<.001). La jornada de estudio, en la que el estudiantado del turno de tarde puntúa significativamente por debajo del turno diurno (<.05); la exigencia académica, en la que quienes consideran tener un nivel muy bajo puntúan significativamente por debajo del bajo y, estos grupos del muy alto (<.05). Así como la calificación docente, en la que los sujetos que se definen como regular y mal se diferencian significativamente del resto $(<.05)$.

El factor "MOT_Asiste2", caracterizado por su asociación a los recursos materiales y humanos, a la metodología docente y al apoyo académico, muestra diferencias significativas en variables tales como el estrato socioeconómico (estrato $5<$ estrato 2 ; <.05), estado civil (divorciado/a < casado/a, m-p soltero/a, unión libre, soltero/a; $<.001)$, ciclo académico $(6<1$; $<.001$ ), exigencia académica (bajo, muy bajo< muy alto; <.05) y calificación docente (regular< mal, muy bien, excelente; bien < excelente; <.05).

Describiendo ejemplos adicionales, se observa igualmente que los motivos para abandonar los estudios definidos como "MOT_Abandono1", asociados a dificultades familiares, individuales, institucionales, académicas y financieras, vienen afectados por variables tales 
como la edad con diferencias significativas (<.05) entre menores de 18 años con el resto de participantes de la muestra; el estrato socioeconómico $(5<6)$; el ciclo académico $(1<2,5,7,8)$; así como la jornada de estudio con asistentes a la jornada de tarde que muestran menor tendencia al abandono que quienes asisten en jornada nocturna y especial (10 a.m.-1 p.m.).

El factor denominado MOT_Abandono2, relacionado con dificultades económicas, familiares y de salud, muestra diferencias significativas $(<.05)$ en las variables edad $(<18 \mathrm{a}$. $<$ todos los demás), estrato socioeconómico $(5<3,6,2)$, fuente de ingresos (beca-subsidio < crto educativo), realización de trabajos (no < sí), ingresos familiares (padres acudientes < recursos propios), ciclo académico $(1<5)$ y jornada de estudio (tarde < nocturno, diurno; especial< nocturno).

\section{Discusión de resultados}

El objetivo de la muestra institucional descrita en este apartado es realizar una caracterización de la población del PI y establecer una relación entre los factores identificados y la variable permanencia estudiantil.

El grupo etario de mayor representación en la institución es de 18 a 20 años, predictor de permanencia favorable para la institución, si se tiene en cuenta que, de acuerdo con estadísticas del MEN, "a nivel nacional ... los estudiantes que ingresan al sistema [con edades mayores] acumulan tasas de deserción ... 17\% más altas ... que ... los estudiantes más jóvenes" (MEN, 2009, p. 92). Asimismo, otro factor de predicción favorable para la institución lo constituye la variable género (es el mayor porcentaje de la población del Politécnico Internacional de género femenino): "Ios hombres alcanzan tasas de deserción más altas frente a las mujeres" (MEN, 2009, p. 93). Dentro de la variable estado civil, el grupo más representativo es el de estudiantado soltero; factor favorable partiendo de estudios previos que caracterizan este estado como ventaja en términos de estabilidad en la vinculación a las instituciones de ES, y menor número de obligaciones de carácter laboral, familiar y social (García de Fanelli, 2012).

En una aproximación a los factores relacionados con la probabilidad de desertar, las dimensiones relacionadas con la permanencia estudiantil han sido divididas de manera que puedan ser relacionadas con las variables que impactan el fenómeno estudiado.

En primer lugar se establece la relación entre los datos recolectados y la variable retención estudiantil, debido al rol institucional en el fortalecimiento de estrategias que permitan la permanencia del estudiantado desde el ingreso al programa hasta su culminación como graduado. La calidad y el perfil del programa, los cambios de institución, el apoyo del personal docente entre otros, forman parte de las variables que deben definirse y evaluarse continuamente al interior de la institución, con el fin de generar oportunidades de mejora que impulsen la oferta institucional de herramientas para que sus estudiantes permanezcan y culminen sus estudios. 
http://doi.org/10.15359/ree.25-1.6

http://www.una.ac.cr/educare

educare@una.ac.cr

En este orden de ideas, el alto puntaje en los cuestionarios de las variables calidad y perfil del programa, señalan su gran relevancia en la decisión estudiantil de continuar vinculándose a la institución. Esta tendencia apunta la necesidad de propender, al interior del establecimiento educativo, por el liderazgo en procesos de crecimiento y renovación continuos basados en el modelo institucional. Estos procesos deben partir del análisis de factores de aula tales como aprendizajes previos, la interacción social y los desempeños de estudiantes, con el fin de generar herramientas para mantener un alto nivel de calidad al interior de los programas.

Relacionado con este análisis se encuentra la constatación de la poca o nula relevancia dada por el estudiantado al factor de aplazamiento de estudios por cambio de institución. La falta de transcendencia se expresa como factor favorable para el PI, en tanto que la mayoría de estudiantes considera de alto interés el acompañamiento del personal docente en el proceso formativo y el apoyo académico brindado por la entidad. Así, se corrobora que la retención, como componente de permanencia estudiantil basada en las políticas y protocolos institucionales, debe tener como prioridad la actualización y renovación de todos los procedimientos relacionados con el acompañamiento de los procesos estudiantiles hasta la culminación del programa.

La segunda dimensión analizada dentro del contexto de la permanencia estudiantil es la persistencia, especificada por Torres Guevara (2012) como la capacidad del estudiantado a nivel académico, social y motivacional para alcanzar sus propias metas educativas, centrándose en sí mismo.

Al establecer una distinción entre retención y persistencia como dimensiones de la institución y del alumnado, respectivamente, se hace necesario identificar posibles conflictos entre las metas, motivación y objetivos de los dos agentes. Fenómenos cuya influencia en estas tres dimensiones ontológicas del estudiante ha sido documentada, incluyen la falta de tiempo para cursar los estudios, los problemas de salud y el embarazo.

Es de destacar cómo la retención y la persistencia se complementan como dimensiones que integran las habilidades y motivaciones de estudiantes con las características y perspectivas institucionales para lograr un mismo fin. Situación ya descrita por Torres Guevara (2012), para quien la permanencia es una condición centrada en las estrategias de retención de la institución y en la persistencia del estudiantado.

La categoría de análisis permanencia estudiantil enmarca los fenómenos de la cotidianidad del estudiantado en su relación con la institución: oportunidad, actualidad de la institución y mejoras orientadas a garantizar su permanencia hasta la obtención del título. La permanencia estudiantil es definida como el lapso de tiempo que toma, desde el ingreso hasta obtener su título profesional (Meléndez 2008, citado en Mendoza et al., 2014). 
Partiendo de esta noción, que implica tener presente las experiencias estudiantiles, tanto desde el punto de vista académico como social, adquiere especial importancia la indagación al interior de las instituciones, sobre características individuales, familiares y académicas que influyen directa o indirectamente en la decisión del alumnado de permanecer en las aulas hasta obtener su titulación. Las variables de este tipo seleccionadas incluyen el nivel de escolaridad de padres, madres, hermanas y hermanos, la ocupación del estudiantado y las prioridades de la familia frente a la actividad académica.

De acuerdo con el MEN (2009), estudiantes cuyas madres poseen un nivel de formación universitaria tienen mayores probabilidades de culminar sus estudios; de igual forma, García de Fanelli (2012) señala que a menores condiciones académicas en el entorno familiar, mayores serán las tasas de deserción, al no considerar la ES como una prioridad del hogar. Otra condición relevante en el contexto de estudio de la permanencia estudiantil es el número de hermanos o hermanas, en tanto "los estudiantes ... [sin] hermanos presentan una tasa de deserción diez puntos por debajo que aquellos que tienen más de cuatro hermanos" (MEN, 2009, p. 89).

En el caso de nuestro estudio, el porcentaje de madres que alcanzaron el nivel de ES es menor que el de las que alcanzaron niveles de educación secundaria; sin embargo, el conteo de respuestas señala resultados favorables relacionados con las prioridades de la familia en cuanto a la educación del estudiantado del PI. En relación con la variable número de hermanos, los resultados permiten enunciar dos factores favorables de retención y permanencia en la institución: un bajo porcentaje de la muestra tiene más de cuatro hermanos o hermanas y un porcentaje considerable de este grupo alcanzaron niveles de ES. En cuanto al factor trabajo de estudiantes, la mayor parte de la muestra indica la combinación de los aspectos laboral y académico; la simultaneidad de ocupaciones (trabajo- estudio) atenúa un factor de riesgo importante de abandono institucional.

\section{Conclusiones}

Atendiendo la identificación de factores que inciden en la retención estudiantil en el PI, observamos su relación con la calidad y el perfil de los programas, así como el apoyo que la institución brinda a su estudiantado para mantenerlo vinculado a los programas académicos.

En relación con la persistencia del estudiantado en la institución, las características que se distinguen se relacionan con la motivación y la valoración del sery de las necesidades particulares, para hacer del paso por la institución un camino agradable que impulse a perseverar hasta la obtención del grado.

Las estrategias para el logro de los objetivos relacionados con la permanencia estudiantil son afines al modelo pedagógico institucionaly a los lineamientosformulados para la disminución 
http://doi.org/10.15359/ree.25-1.6

http://www.una.ac.cr/educare

educare@una.ac.cr

del abandono académico. Dichas estrategias deben orientarse, de manera interactiva y cotidiana, en los macro procesos de gestión universitaria, gestión académica, bienestar institucional, apoyos financieros y apoyos personales, como evidencia la planificación de acciones tutoriales enfocadas hacia la carrera (Clerici y Da Re, 2019). A este respecto, se insiste en la importancia de la creación e implementación, a nivel institucional, de estrategias de evaluación del impacto de los planes desarrollados, con el fin de garantizar la efectividad de los procesos. Pueden asumirse, por tanto, algunas implicaciones, tanto para la gestión institucional, como para el marco de políticas inclusivas que favorezcan la atención al estudiantado, fundamentalmente en aquellos casos que pueden derivarse en vulnerables.

Como proyección a este trabajo de investigación se sugiere reconocer y caracterizar la realidad de la situación de permanencia estudiantil en educación superior dentro de cada contexto particular, con el fin de generar estrategias pertinentes a las relaciones y los factores identificados. Dichas estrategias deberán implementarse a partir del análisis de la realidad de cada institución educativa, pues el perfil del estudiantado puede variar en función de esta misma.

\section{Limitaciones}

El estudio realizado se ha focalizado en una institución educativa, en un caso, por lo que los supuestos de generalización de los resultados presentados pueden ser útiles para otros casos o instituciones, en la medida que dispongan con caraterísticas similares a las presentadas. Hay que tener presente que este trabajo se enmarca en uno más genérico que incluye el papel del personal docente y del personal de gestión institucional, conformando un modo de triangulación de fuentes de información, que no ha sido utilizada en el presente análisis. Por ello, se reconocen, como limitaciones del estudio, la recolección de información demandada a estudiantes sin incluir personal administrativo y profesorado de la institución. De igual manera, la obtención de información complementaria mediante el uso de instrumentos adicionales de recolección (entrevistas, grupos focales) que consideren la profundización desde el análisis cualitativo, integrando la información cuantitativa. Asimismo, una extensión de la investigación mediante la incorporación de la diversidad de tipologías institucionales de educación superior permitiría incidir sobre políticas globales que mejoren los niveles de calidad y sostenimiento institucional en cuanto a la retención, persistencia y permanencia del estudiantado en dichas instituciones.

\section{Declaración de Material complementario}

Este artículo tiene disponible, como material complementario:

-La versión preprint del artículo en https://doi.org/10.5281/zenodo.3420895 
http://doi.org/10.15359/ree.25-1.6

http://www.una.ac.cr/educare educare@una.ac.cr

\section{Referencias}

Apaza, E. y Huamán, F. (2012). Factores determinantes que inciden en la deserción de los estudiantes universitarios. Revista Apuntes Universitarios, 2(1), 77-86 https:// apuntesuniversitarios.upeu.edu.pe/index.php/revapuntes/article/view/306/pdf

Báez, C. P. Higuera Rojas, R. Ortega Tobar, N. y Quintero Oviedo, J. A. (2010). Persistencia y graduación: Hacia un modelo de retención estudiantil para las instituciones de educación superior en Colombia. Los programas de fomento de la permanencia en la Universidad de La Sabana: Pilotaje de un instrumento [Tesis de maestría]. Universidad de la Sabana, Colombia. https://intellectum.unisabana.edu.co/handle/10818/2532

Barrero, F. (2015). Investigación en deserción estudiantil universitaria: Educación, cultura y significados. Revista Educación y desarrollo social, 9(2), 86-101. https://doi.org/10.18359/ reds.948

Clerici, R. y Da Re, L. (2019). Evaluación de la eficacia de un programa de tutoría formativa. Revista de Investigación Educativa, 37(1), 39-56. https://doi.org/10.6018/rie.37.1.322331

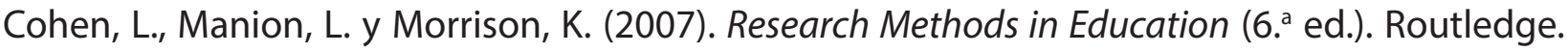
https://doi.org/10.4324/9780203029053

Fernández-Hileman, M. del R., Corengia, Á. y Durand, J. (2014). Deserción y retención universitaria: Una discusión bibliográfica. Pensando Psicología/, 10(17), 85-96. https://doi.org/10.16925/ pe.v10i17.787

García de Fanelli, A. M. (2012). Acceso, abandono y graduación en la educación superior argentina. SITEAL.

González-Ramírez, T. y Pedraza-Navarro, I. (2017). Variables sociofamiliares asociadas al abandono de los estudios universitarios. Educatio Siglo XXI, 35(2), 365-388. https://doi. org/10.6018/j/298651

Himmel, E. (2002). Modelos de análisis de la deserción en la educación superior. Calidad en educación, 17, 91-108. https://doi.org/10.31619/caledu.n17.409

Íñiguez, T., Elboj Saso, C. y Valero Errazu, D. (2016). La Universidad del Espacio Europeo de Educación Superior ante el abandono de los estudios de grado. Causas y propuestas estratégicas de prevención. Educar, 52(2), 285-313. https://doi.org/10.5565/rev/educar.674

Korantowicz, E. y Nizinska, A. (2013). How students 'stay the course': Retention practices in higher education. Studies in the Education of Adults, 45(2), 135-47. https://doi.org/10.1080 $\underline{\text { /02660830.2013.11661647 }}$ 
http://doi.org/10.15359/ree.25-1.6

http://www.una.ac.cr/educare

educare@una.ac.cr

Meléndez Surmay, R. y Meriño Mendoza, D. (2008). Estudio sobre deserción y permanencia académica en la Facultad de Ingeniería de la Universidad de la Guajira desde el II PA 2005 haste el II PA 2007 [Tesis de pregrado]. Universidad de la Guajira, Colombia. https://docplayer. es/12234104-Estudio-sobre-desercion-y-permanencia-academica-en-la-facultad-deingenieria-de-la-universidad-de-la-guajira-desde-el-ii-pa-2005-hasta-el-ii-pa-2007.html

Mendoza Gutiérrez, L. Mendoza Rubio, U.y Romero Meléndez, D. (2014). Permanencia académica: Una preocupación de las instituciones de educación superior. Escenarios, 12(2), 130-137. https://doi.org/10.15665/esc.v12i2.320

Ministerio de Educación Nacional. (2009). Deserción estudiantil en la educación superior colombiana. Metodología de seguimiento, diagnóstico y elementos para su prevención. Autor. https://www.mineducacion.gov.co/portal/micrositios-superior/PublicacionesEducacion-Superior/356271:Desercion-estudiantil-en-la-educacion-superior-colombiana

Ministerio de Educación Nacional (2015). Estrategias para la permanencia en educación superior: Experiencias significativas. Autor. https://www.mineducacion.gov.co/1759/ articles-356276 recurso.pdf

Mucchielli, A. (2001). Diccionario de métodos cualitativos en ciencias humanas y sociales. Editorial Síntesis.

Munizaga, F., Cifuentes, M. B.y Beltrán, A. (2018). Retención y abandono estudiantil en la educación superior universitaria en América Latina y el Caribe: Una revisión sistemática. Archivos Analíticos de Políticas Educativas, 26(61), 1-36. https://doi.org/10.14507/epaa.26.3348

Páramo, G. J. y Correa, C. A. (1999). Deserción estudiantil universitaria. Conceptualización. Revista Universidad Eafit, 35(114), 65-78. http://publicaciones.eafit.edu.co/index.php/revistauniversidad-eafit/article/view/1075

Pineda-Báez, C., Pedraza-Ortiz, A.y Moreno, I.D. (2011). Efectividad de las estrategias de retención universitaria: La función del docente. Educación y educadores, 14(1), 119-135. http://www. scielo.org.co/scielo.php?script=sci abstract\&pid=S0123-12942011000100008\&lng=en\& $\underline{\mathrm{nrm}=\mathrm{i} \& \text { \&tlng=es }}$

Rosado, M. Á. (2006). Metodología de investigación y evaluación. Trillas.

Stake, R. E. (2007). Investigación con estudio de casos (4. ${ }^{\mathrm{a}}$ ed.). Morata.

Tinto, V. (1989). Definir la deserción: Una cuestión de perspectiva. Revista de Educación Superior, 18(71), 33-51. http://publicaciones.anuies.mx/revista/71/1/3/es/definir-la-desercion-unacuestion-de-perspectiva 
http://doi.org/10.15359/ree.25-1.6 http://www.una.ac.cr/educare educare@una.ac.cr

Tinto, V. (2010). From theory to action: Exploring the institutional conditions for student retention. Higher Education: Handbook of Theory and Research, 25, 51-89. https://doi. org/10.1007/978-90-481-8598-6 2

Torrado, M. (2012). El fenómeno del abandono en la UB: El caso de ciencias experimentales [Tesis doctoral]. Universitat de Barcelona, España. http://www.tdx.cat/handle/10803/134955

Torres Guevara, L. E. (2012). Retención estudiantil en la educación superior: Revisión de la literatura y elementos de un modelo para el contexto colombiano. Editorial Pontificia Universidad Javeriana.

Tuero, E., Cervero, A., Esteban, M., y Bernardo, A. (2018). Por qué abandonan los alumnos universitarios? Variables de influencia en el planteamiento y consolidación del abandono. Educación XX1, 21(2), 131-154. https://doi.org/10.5944/educxx1.20066

Velásquez, M., Posada, M., Gómez, D. N., López, N., Vallejo, F., Ramírez, P. A., Hernández, C. y Vallejo, A. (2011). Acciones para favorecer la permanencia. Universidad de Antioquia. 2011. Colombia En I Congresos CLABES (pp. 1-9). Universidad Nacional Autónoma de Nicaragua. https://revistas.utp.ac.pa/index.php/clabes/issue/view/62

Yin, R. K. (2009). Case study research. Design and methods (4. ${ }^{\text {a }}$ ed.). Sage. 